\title{
Activation of the ERK1/2 Mitogen-Activated Protein Kinase Cascade by Dentin Matrix Protein 1 Promotes Osteoblast Differentiation
}

\author{
Asha Eapen $^{\mathrm{a}} \quad$ Amsaveni Ramachandran $^{\mathrm{a}} \quad$ Jitesh Pratap $^{\mathrm{b}} \quad$ Anne George $^{\mathrm{a}}$ \\ ${ }^{a}$ Brodie Tooth Development Genetics and Regenerative Medicine Research Laboratory, University of \\ Illinois at Chicago, and ${ }^{\mathrm{b}}$ Anatomy and Cell Biology, Rush University Medical Center, Chicago, III., USA
}

\section{Key Words}

Dentin matrix protein $1 \cdot$ ERK1/2 MAP kinase $\cdot$ Runx2 $\cdot$

$\mathrm{RGD} \cdot$ Osteoblast differentiation

\begin{abstract}
DMP1 has been shown to play many roles in osteogenesis. We recently demonstrated that calcium-mediated stress kinase activation by DMP1 leads to osteoblast differentiation. In this study we demonstrate that DMP1 can also activate the extracellular signal-regulated kinase (ERK)-MAPK pathway. This activation was mediated through the RGD integrinbinding domain in DMP1. Further, we demonstrate that Runx2, an essential transcription factor, is stimulated by the ERK-MAPK pathway.

Copyright $\odot 2011$ S. Karger AG, Basel
\end{abstract}

\section{Introduction}

MAP kinases function in many cell types; they are regulated by a diverse group of extracellular stimuli and mediate a variety of cellular responses [Cowley et al., 1994]. Published reports suggest that the duration and intensity of mitogen-activated protein kinase (MAPK) activation can profoundly influence the biological re-

\section{KARGER}

Fax +4161306 1234

E-Mail karger@karger.ch

www.karger.com (c) 2011 S. Karger AG, Basel

Accessible online at: www.karger.com/cto sponse observed [Cowley et al., 1994; Lee et al., 2000]. The extracellular signal-regulated kinase (ERK)-MAPK pathway has important functions in the differentiation of postmitotic cells. In addition, this pathway can regulate the activity of several lineage-specific transcription factors. In bone, the ERK-MAPK pathway is a major conduit for conveying information about the extracellular environment to the nucleus and it has been implicated in the response of bone to a variety of signals, including hormone/growth factor stimulation, extracellular matrix-integrin binding, and mechanical loading [Ge et al., 2007].

In a recent study we demonstrated that dental matrix protein 1 (DMP1) stimulation supports a stimulatory role

\section{Abbreviations used in this paper}

2APB 2-aminoethoxydiphenyl borate

BAPTA-AM 1,2-bis-(o-aminophenoxy)-ethane-N,N, $\mathrm{N}^{\prime}, \mathrm{N}^{\prime}$ tetra-acetic acid, tetra-acetoxymethyl ester

DMP1 dentin matrix protein1

ERK1/2 extracellular signal-regulated kinase 1/2

IP3 inositol triphosphate

MAPK mitogen activated protein kinase

RGD arginine-glycine-aspartic acid

RT-qPCR quantitative real-time PCR 
in osteoblast differentiation [Eapen et al., 2010]. Quantitative PCR results showed that Runx 2 and osteocalcin mRNA expression levels were elevated by DMP1 treatment in MC3T3-E1 cell cultures. Four hour exposures with a p38 kinase inhibitor (SB203580) reduced the DMP1 elevated Runx2 and osteocalcin mRNA expression levels, suggesting the involvement of p38 kinase in the signal transduction pathway. Western blot analyses using an antibody that recognizes the phosphorylated (i.e. activated) form of p38 kinase showed rapid elevated levels upon treatment with DMP1. Experiments with an inositol triphosphate (IP3)R antagonist (2-aminoethoxydiphenyl borate; 2APB) or a PLC inhibitor (U73122) showed that these elevated intracellular calcium transients are the result of calcium release from endoplasmic reticulum stores. Treatment with BAPTA-AM resulted in the attenuation of p38 phosphorylation, confirming the role of $\mathrm{Ca}^{2+}$ in downstream signaling. The addition of the calphostin C control clearly indicates that a PLC conversion of IP4 into IP3 and DAG (which would activate PKC) is involved in this DMP1-mediated calcium release [Eapen et al., 2010].

In this study we sought to gain insight into additional signaling by other members of the MAP kinase family. We provide evidence of the activation of the ERK-MAPK cascade in preosteoblasts in response to DMP1 stimulation. Thus, DMP1 stimulation can regulate osteoblast differentiation by activating both the ERK and the p38 MAP kinase signaling pathways in preosteoblasts.

\section{Materials and Methods}

Expression and Purification of DMP-1

The recombinant DMP1 protein was expressed in Escherichia coli as published earlier [Srinivasan et al., 1999].

\section{Cell Culture}

Mouse calvarial preosteoblast MC3T3-E1 cells (a kind gift from Dr. R.T. Franceschi of the University of Michigan) were cultured in $\alpha$-MEM medium supplemented with $10 \%$ FBS and $1 \%$ penicillin-streptomycin. The cells were seeded in 6-well plates and allowed to proliferate until $70 \%$ confluence had been attained. The medium was changed every 2 days. 12 to $16 \mathrm{~h}$ before the start of the experiment the cells were cultured in $\alpha$-MEM medium supplemented with $1 \%$ FBS (basal medium). These cells were then stimulated with $250 \mathrm{ng} / \mathrm{ml} \mathrm{rDMP} 1$. The treated cells were then trypsinized and RNA was extracted for RT-qPCR. Total proteins were extracted at $30 \mathrm{~min}, 1 \mathrm{~h}$, and $2 \mathrm{~h}$, respectively.

\section{Quantitative Real-Time PCR}

RNA was extracted according to the manufacturer's recommended protocol using Trizol (Invitrogen). Quantitative real- time PCR (RT-qPCR) was performed with DNase I (Promega)treated RNA as published earlier [Eapen et al., 2010]. Expression of osteocalcin, Runx2, and GAPDH transcripts was analyzed by qPCR during its linear phase.

\section{Detection of Protein Phosphorylation by Western Blot}

Analysis

Total proteins were extracted from rDMP1-stimulated MC3T3-E1 cells using M-PER reagent (Pierce). 35 micrograms of total proteins were resolved on a $10 \%$ SDS-polyacrylamide gel under reducing conditions. After electrophoresis, the proteins were electro-transferred onto nitrocellulose membrane (BioRad Laboratories), blocked with 5\% nonfat milk, and probed with anti-ERK1/2 (1:500) (Santa Cruz), anti-phospho-ERK1/2 (1:500) (Santa Cruz), anti-MEK1/2 (1:500) (Cell Signaling), antiphospho-MEK1/2 (1:500) (Cell Signaling), or anti-Runx2 (1:500) (Abcam) for $16 \mathrm{~h}$ at $4^{\circ} \mathrm{C}$. Blots were then incubated with HRPconjugated goat anti-rabbit IgG secondary antibody (Chemicon International) or HRP-conjugated goat anti-mouse IgG secondary antibody (Sigma). They were washed 3 times with PBS containing $0.05 \%$ Tween 20 and once with PBS. The bands were visualized by the ECL-Western blot reagent (Perkin-Elmer Life Sciences). Each membrane was then carefully washed, treated for 10 min with a stripping buffer (Pierce), and washed with PBS. Western blot analysis was performed with mouse anti-tubulin antibody (1:10,000) (Sigma) and HRP-conjugated goat anti-mouse IgG secondary antibody.

Treatment of Cells with PD98059 and Blocking with

Arginine-Glycine-Aspartic Acid Peptide

Cells were cultured as described above and treated with $15 \mu \mathrm{M}$ PD98059 (Biomol) - an inhibitor specific for ERK1/2 MAP kinase. PD98059 was added to the rDMP1 containing basal media and preincubated for $30 \mathrm{~min}$ at $37^{\circ} \mathrm{C}$ prior to the start of the experiment. Total RNA and cell lysates were harvested at various time points and analyzed by qPCR and Western blotting performed as described above.

For the arginine-glycine-aspartic acid (RGD) blocking experiment, MC3T3-E1 cells were incubated with the RGD-blocking peptide $(2 \mathrm{~mm})$ for $60 \mathrm{~min}$. The cells were then stimulated with rDMP1 for $1 \mathrm{~h}$ and total proteins were extracted. Western blot was performed to determine the phosphorylation of ERK1/2.

\section{Immunofluorescence}

Activation of ERK1/2 signaling by DMP1 was analyzed by immunofluorescence. MC3T3-E1 cells were seeded on glass slides and treated with $250 \mathrm{ng} / \mathrm{ml} \mathrm{rDMP} 1$ for $30 \mathrm{~min}, 1 \mathrm{~h}$, and $2 \mathrm{~h}$. The cells were subsequently washed with $1 \times$ PBS fixed with $4 \%$ paraformaldehyde for $30 \mathrm{~min}$. Fixed cells were then rinsed thrice with $1 \times$ PBS and permeabilized with $0.05 \%$ Triton X-100 in PBS for 20 min. The cells were then washed with PBS and blocked with $5 \%$ BSA in PBS for $1 \mathrm{~h}$. After blocking, the cells were incubated overnight with anti-phospho-ERK1/2 (1:100) (Santa Cruz) followed by $1 \mathrm{~h}$ of incubation with a fluorescein-conjugated goat anti-rabbit IgG (1:100) (Sigma). After washing with PBS the cover glass was mounted using mounting media (Vector shield), and labeling was detected with an Axio Observer D1 fluorescence microscope (Zeiss) equipped with Axiovision imaging software (Zeiss). 


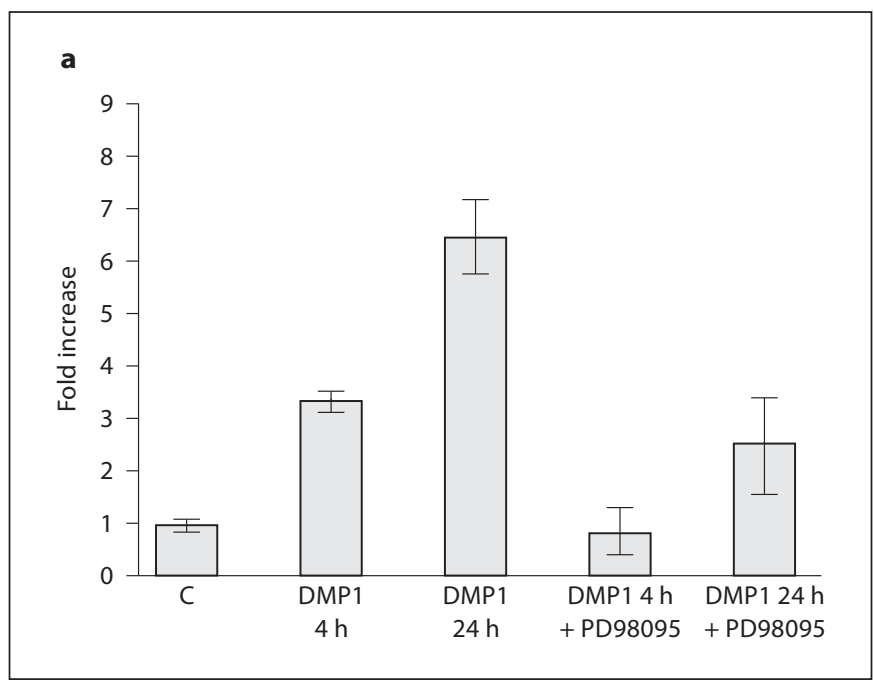

Fig. 1. a Effect of DMP1 on Runx2 mRNA expression and its abrogation in the presence of ERK inhibitor PD98095. MC3T3-E1 cells were either left untreated (control) or starved for $24 \mathrm{~h}$ prior to stimulation with $250 \mathrm{ng} / \mathrm{ml} \mathrm{rDMP1}$ or with PD98095 and rDMP1 in basal medium for 4 and $24 \mathrm{~h}$. Total RNA was isolated, subjected to real-time PCR, and analyzed for the expression of Runx2. These

\section{Results}

DMP1 Stimulation Induces the Expression of Runx2

MC3T3-E1 cells stimulated with DMP1 can activate the gene expression of Runx2. The results in figure 1a show a 3- and 6-fold increase in Runx2 gene expression with DMP1 stimulation. In the presence of ERK1/2 inhibitor PD98059, these effects were abrogated. These results demonstrate that DMP1-mediated ERK1/2 activation is required for osteoblast differentiation. Western blot analysis performed on total cell extracts after DMP1 stimulation confirmed the upregulation of Runx2 protein levels (fig. 1b).

\section{DMP1 Stimulation Induces ERK1/2 Activation}

We next explored the phosphorylation state of ERK1/2 mitogen-activated kinase in preosteoblasts stimulated by DMP1. Western blot analysis performed on DMP1-stimulated cells at $30 \mathrm{~min}, 1 \mathrm{~h}$, and $2 \mathrm{~h}$ showed sustained activation from $30 \mathrm{~min}$ to $2 \mathrm{~h}$ (fig. 2a). An increase in phosphorylation at Thr 202/204 demonstrates the activation of ERK1/2 in response to DMP1 stimulation. When cells were treated with the ERK1/2 inhibitor PD98059, this activation was suppressed (fig. 2c), indicating that induction of osteoblast differentiation depends on ERK activation.

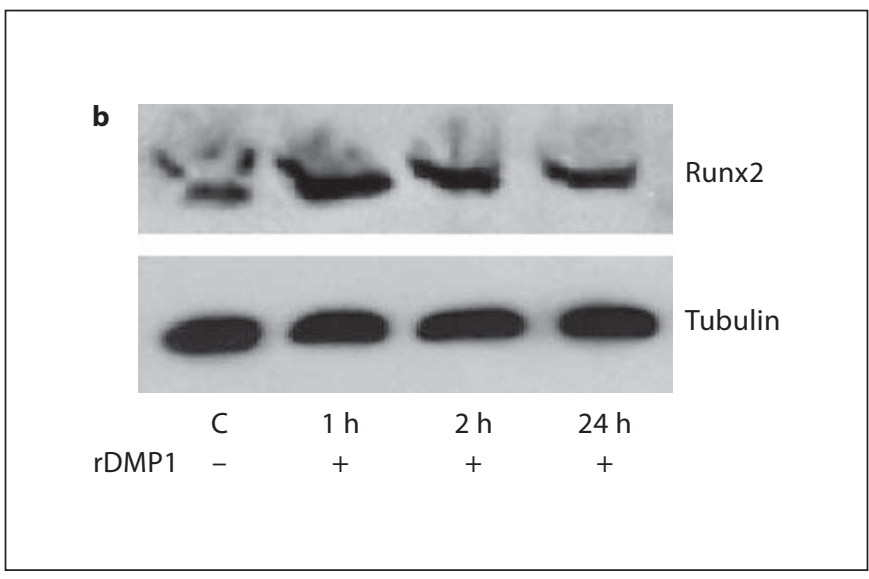

results were normalized with the loading control GAPDH. Experiments were performed in triplicate. b Effect of DMP1 on Runx2 protein expression. MC3T3-E1 cells were either left untreated (control) or starved for $24 \mathrm{~h}$ prior to stimulation with $250 \mathrm{ng} / \mathrm{ml}$ rDMP1 for 1, 2, and $24 \mathrm{~h}$. Total protein was then isolated and subjected to Western blot analysis using mouse anti-Runx 2 antibody.

\section{DMP1-Mediated ERK1/2 Activation Occurs through $M E K 1 / 2$}

To test whether activation of MEK, an upstream regulator, is required for ERK activation, Western blot analysis was performed. The results in figure $2 \mathrm{~b}$ show the activation of MEK1/2 in response to DMP1 stimulation. This data suggests that the activation of MEK is more pronounced in DMP1-stimulated cells when compared with the control. MEK1/2 activation is characterized by activation by phosphorylation at 2 activation loop residues, i.e. Ser 217 and 221.

\section{DMP1-Mediated ERK1/2 Activation in Preosteoblasts Is Mediated through the RGD Integrin-Binding Domain}

DMP1 contains an RGD integrin-binding domain; therefore, we investigated the role of integrin-mediated intracellular signaling. An RGD-blocking peptide was used to study its effect on the activation of ERK1/2. The Western blot analysis presented in figure $2 \mathrm{~d}$ shows that blocking the integrin-binding domain with an RGD peptide did suppress ERK1/2 activation. The ability of DMP1 to suppress ERK1/2 activation in the presence of the RGD peptide suggests a role for integrins in ERK1/2 activation. 


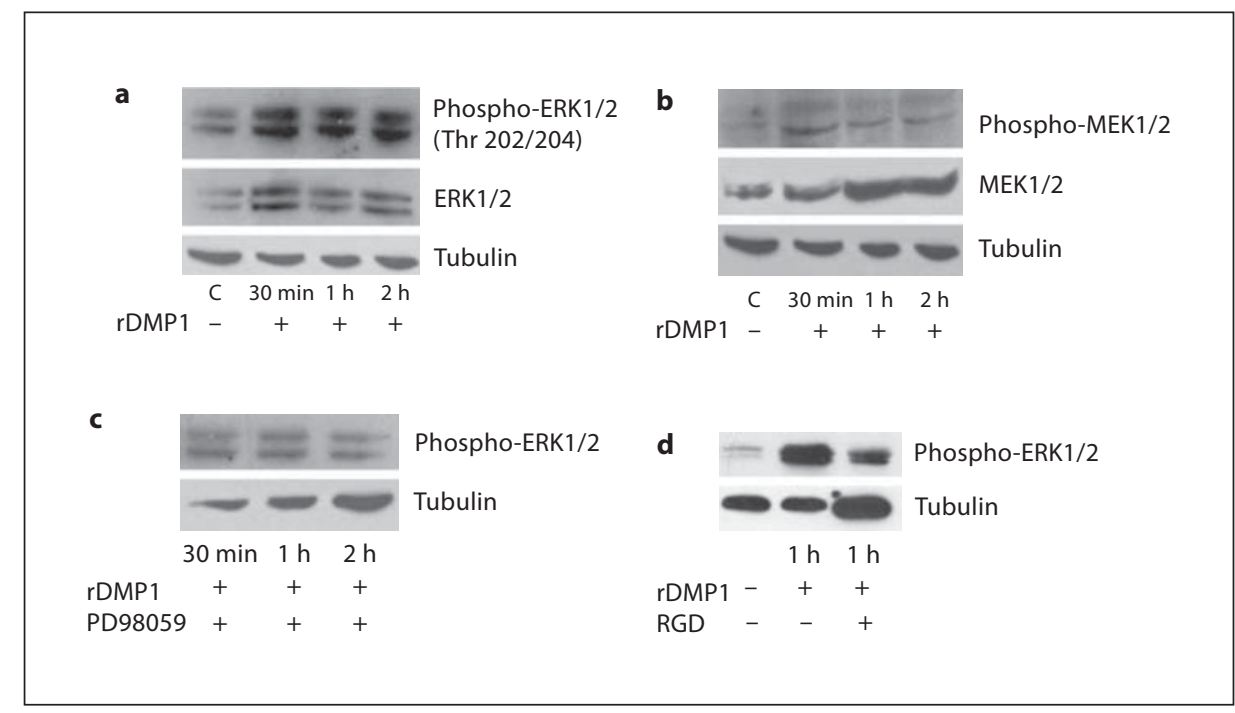

Fig. 2. a DMP1 stimulates phosphorylation of the ERK1/2 MAP kinase in MC3T3-E1 cells. MC3T3-E1 cells in basal medium were untreated (control) or treated with rDMP1 $(250 \mathrm{ng} / \mathrm{ml})$ for 30 $\min , 1 \mathrm{~h}$, and $2 \mathrm{~h}$. Cell lysates were harvested and subjected to SDS-PAGE and Western blot analysis performed with phosphoERK1/2 antibody. The blots were stripped and probed for total ERK1/2. Equal loading of the proteins was confirmed by stripping the blot and then probing it with tubulin. b DMP1 stimulates phosphorylation of the MEK1/2 MAP kinase in MC3T3-E1 cells. MC3T3-E1 cells in basal medium were untreated (control) or treated with rDMP1 $(250 \mathrm{ng} / \mathrm{ml})$ for $30 \mathrm{~min}, 1 \mathrm{~h}$, and $2 \mathrm{~h}$. Cell lysates were harvested and subjected to SDS-PAGE and Western blot analysis performed with phospho-MEK1/2 antibody. The blots were stripped and probed for total MEK1/2. Tubulin was used as a loading control. c DMP1-stimulated phosphorylation of ERK1/2 is abrogated in the presence of PD98059. Cell lysates obtained from control and MC3T3-E1 cells treated with the ERK1/2 MAP kinase inhibitor PD98059 and then stimulated with DMP1 for $30 \mathrm{~min}, 1 \mathrm{~h}$, and $2 \mathrm{~h}$ were immunoblotted with phosphospecific ERK1/2 antibody. Tubulin was used as a loading control. d DMP1-stimulated phosphorylation of ERK1/2 is abrogated in the presence of an RGD-blocking peptide. MC3T3-E1 cells were incubated with or without an RGD-blocking peptide and then stimulated with DMP1 for $1 \mathrm{~h}$ and immunoblotted with phospho-specific ERK1/2 antibody. Tubulin was used as a loading control.
Translocation of Phospho-ERK1/2 to the Nucleus of Preosteoblasts upon DMP1 Stimulation

Confocal analysis of DMP1-stimulated MC3T3-E1 cells stained for phospho-ERK1/2 showed nuclear localization within $30 \mathrm{~min}$ and intense staining by $2 \mathrm{~h}$ (fig. 3), while unstimulated cells showed diffuse cytoplasmic staining. This is consistent with the immunoblotting analysis shown in figure 2a which demonstrates an increase in the phosphorylation of ERK1/2 in MC3T3-E1 cells.

\section{Discussion}

The ability of DMP1 to induce the phosphorylation and activation of ERK1/2 represents an important signaling mechanism in the osteoblast maturation process. In this study we identified that the stimulation of preosteoblasts by DMP1 leads to the activation of ERK1/2. In our previous study, we demonstrated that DMP1 stimulation induces the activation of p38 MAPK and interestingly, p38 activation is required for all phases of osteoblast differentiation [Eapen et al., 2010].

The ERK pathway is one of the best studied MAPK pathways in mammals [Dhillon et al., 2007; Gayer et al., 2010]. ERK1/2 activation regulates proliferation, differentiation, survival, migration, angiogenesis, and even chromatin remodeling through the phosphorylation of both cytoplasmic and nuclear targets including phosphatases, transcriptional factors, and cytoskeletal proteins. In the canonical ERK1/2 pathway, receptor tyrosine kinases are activated by specific ligands and trigger guanosine triphosphate (GTP) loading of the Ras protein which can then recruit the Raf kinases (A-Raf, B-Raf, and cRaf) [Robertson et al., 2010]. These kinases consecutively phosphorylate and activate MEK (MEK1 and MEK2), ultimately leading to the activation of ERK1/2. In addition to the canonical pathway, ERK1/2 has been shown to be activated by a variety of pathways depending on the in- 

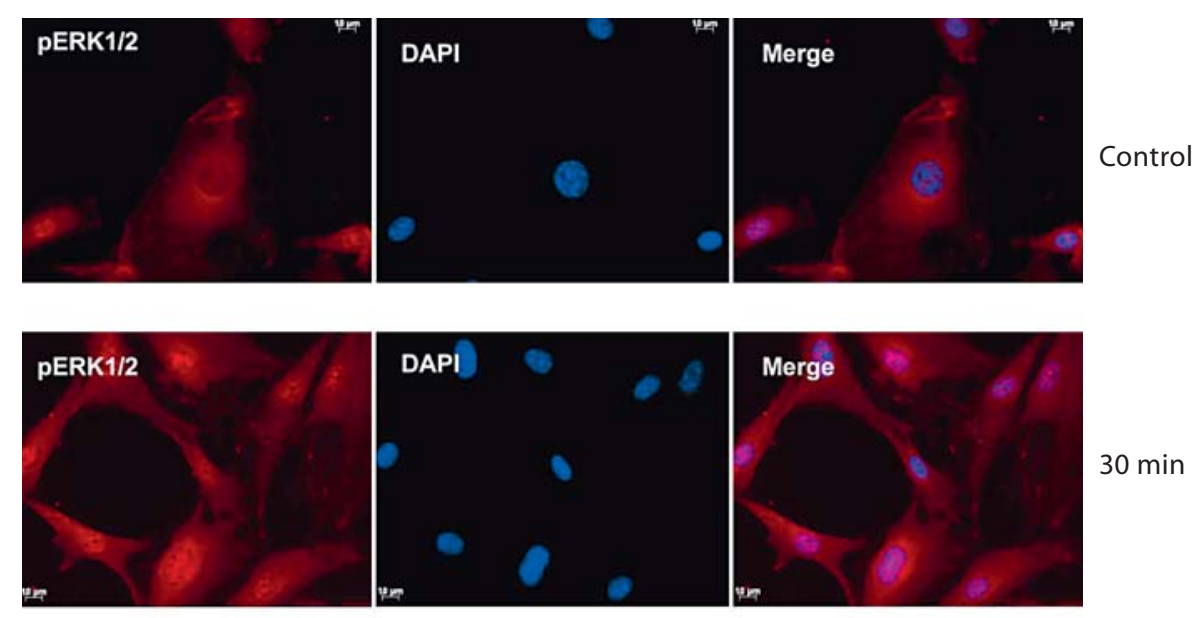

Fig. 3. DMP1 stimulation leads to the accumulation of phospho-ERK1/2 in the nucleus of MC3T3-E1 cells. MC3T3 cells were incubated with anti-phospho-ERK antibody after stimulation with DMP1 for $30 \mathrm{~min}, 1 \mathrm{~h}$, and $2 \mathrm{~h}$ followed by incubation with secondary antibody, and they were subjected to confocal imaging. The confocal image shows the subcellular localization of phospho-ERK1/2. It is important to note that in the control unstimulated cells phospho-ERK1/2 was predominantly cytoplasmic while the intensity of the nuclear staining increased from $30 \mathrm{~min}$ to $2 \mathrm{~h}$. Scale bars $=10 \mu \mathrm{m}$.
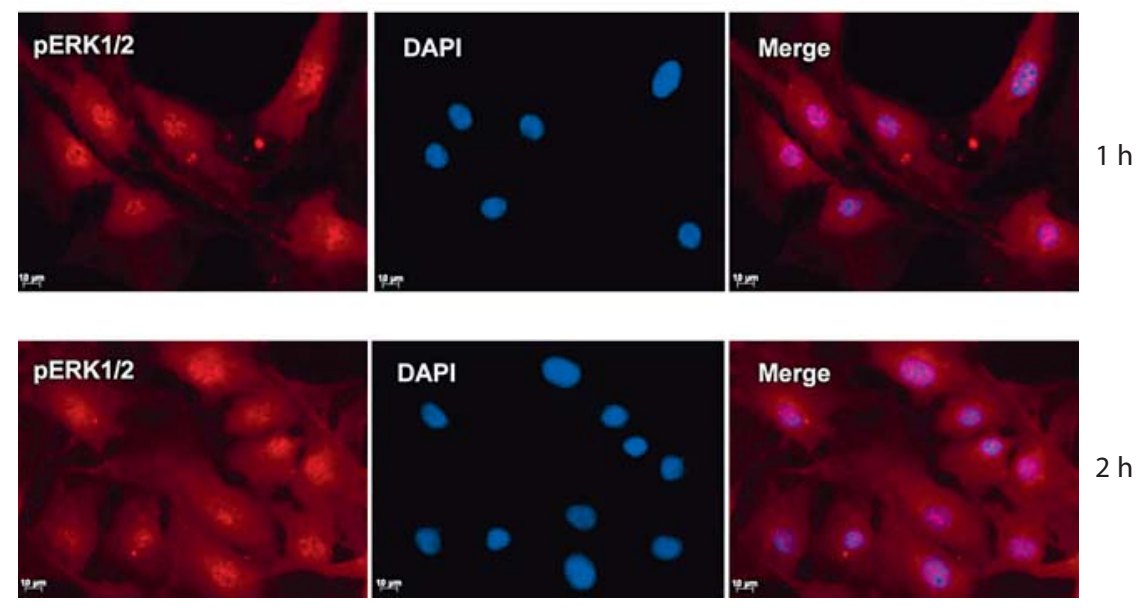

dividual ligand, cell surface receptor, and cell type. Although MAP kinases participate in different signaling pathways, they may be functionally related and coactivated in response to certain stimuli.

DMP1 is a noncollagenous protein present in the bone and dentin matrix [George et al., 1993; D'Souza et al., 1997; MacDougall et al., 1998]. We showed earlier that DMP1 is also specifically localized in the nucleus of differentiating osteoblasts and odontoblasts [Narayanan et al., 2003]. Translocation of DMP1 from the extracellular matrix is facilitated by the plasma membrane-localized GRP78 [Ravindran et al., 2008]. An interesting observation was that the internalization of DMP1 results in stored $\mathrm{Ca}^{2+}$ release and the activation of p38 MAP kinase [Eapen et al., 2010]. Knockout models of DMP1 have shown predominant skeletal defects confirming a role for DMP1 in osteoblast differentiation and mineralization [Feng et al., 2006].

DMP1 Activates ERK1/2 MAPK

Signaling
In this study we demonstrated that DMP1 induces ERK activation leading to osteoblast differentiation as the expression of osteocalcin and Runx 2 could be abrogated by PD98059, a chemical inhibitor of ERK1/2 kinase. Several studies have shown that ERK1/2 activation stimulates Runx 2 activation and osteoblast differentiation [Ge et al., 2007; Raucci et al., 2008; Franceschi et al., 2009; Jun et al., 2010]. DMP1 contains an integrin-binding RGD domain. We demonstrated in this study that the phosphorylation state of ERK1/2 is mediated through integrins as blocking the RGD domain with a specific blocking peptide abrogated ERK phosphorylation. Upstream regulators of ERK1/2 are Raf and MEK1/2. Results from this study showed activation of MEK1/2 in osteoblasts stimulated with DMP1 when compared with control cells, indicating that ERK activation mediated by DMP1 is mediated through the MEK/ERK signaling pathway.

In conclusion, our findings from this study establish the molecular connection between the MEK/ERK signal 
transduction pathway and osteoblast differentiation mediated by the integrin-binding RGD domain of DMP1. Overall, the data suggests the possibility of cross talk between the p38 MAPK and ERK pathways facilitating osteoblast differentiation.

\section{Acknowledgements}

This work was supported by National Institutes of Health Grant DE 11657 and by the Brodie Endowment Fund.

\section{References}

Cowley, S., H. Paterson, P. Kemp, C.J. Marshall (1994) Activation of MAP kinase kinase is necessary and sufficient for PC12 differentiation and for transformation of NIH 3T3 cells. Cell 77: 841-852.

Dhillon, A.S., S. Hagan, O. Rath, W. Kolch (2007) MAP kinase signalling pathways in cancer. Oncogene 26: 3279-3290.

D'Souza, R.N., A. Cavender, G. Sunavala, J. Alvarez, T. Ohshima, A.B. Kulkarni, M. MacDougall (1997) Gene expression patterns of murine dentin matrix protein 1 (Dmpl) and dentin sialophosphoprotein (DSPP) suggest distinct developmental functions in vivo. J Bone Miner Res 12: 2040-2049.

Eapen, A.S., P. Sundivakkam, Y. Song, S. Ravindran, A. Ramachandran, C. Tiruppathi, A. George (2010) Calcium-mediated stress kinase activation by DMP1 promotes osteoblast differentiation. J Biol Chem 285: 36339_ 36351.

Feng, J.Q., L.M. Ward, S. Liu, Y. Lu, Y. Xie, B. Yuan, X. Yu, F. Rauch, S.I. Davis, S. Zhang, H. Rios, M.K. Drezner, L.D. Quarles, L.F. Bonewald, K.E. White (2006) Loss of DMP1 causes rickets and osteomalacia and identifies a role for osteocytes in mineral metabolism. Nat Genet 38: 1310-1315.
Franceschi, R.T., C. Ge, G. Xiao, H. Roca, D. Jiang (2009) Transcriptional regulation of osteoblasts. Cells Tissues Organs 189: 144152.

Gayer, C.P., D.H. Craig, T.L. Flanigan, T.D. Reed, D.E. Cress, M.D. Basson (2010) ERK regulates strain-induced migration and proliferation from different subcellular locations. J Cell Biochem 109: 711-725.

Ge, C., G. Xiao, D. Jiang, R.T. Franceschi (2007) Critical role of the extracellular signal-regulated kinase-MAPK pathway in osteoblast differentiation and skeletal development. J Cell Biol 176: 709-718.

George, A., B. Sabsay, P.A. Simonian, A. Veis (1993) Characterization of a novel dentin matrix acidic phosphoprotein: implications for induction of biomineralization. J Biol Chem 268: 12624-12630.

Jun, J.H., W.J. Yoon, S.B. Seo, K.M. Woo, G.S. Kim, H.M. Ryoo, J.H. Baek (2010 ) BMP2activated Erk/MAP kinase stabilizes Runx2 by increasing p300 levels and histone acetyl transferase activity. J Biol Chem 285: 3641036419.

Lee, S.W., L. Fang, M. Igarashi, T. Ouchi, K.P. Lu, S.A. Aaronson (2000) Sustained activation of Ras/Raf/mitogen-activated protein kinase cascade by the tumor suppressor p53. Proc Natl Acad Sci USA 97: 8302-8305.
MacDougall, M., T.T. Gu, X. Luan, D. Simmons, J. Chen (1998) Identification of a novel isoform of mouse dentin matrix protein 1: spatial expression in mineralized tissues. J Bone Miner Res 13: 422-431.

Narayanan, K., A. Ramachandran, J. Hao, G. He, K.W. Park, M. Cho, A. George (2003) Dual functional roles of dentin matrix protein 1 : implications in biomineralization and gene transcription by activation of intracellular $\mathrm{Ca}^{2+}$ store. J Biol Chem 278: 17500-17508.

Raucci, A., P. Bellosta, R. Grassi, C. Basilico, A. Mansukhani (2008) Osteoblast proliferation or differentiation is regulated by relative strengths of opposing signaling pathways. J Cell Physiol 215: 442-451.

Ravindran, S., K. Narayanan, A.S. Eapen, J. Hao, A. Ramachandran, S. Blond, A. George (2008) Endoplasmic reticulum chaperone protein GRP-78 mediates endocytosis of dentin matrix protein 1. J Biol Chem 283: 29658-29670.

Robertson, B.W., L. Bonsal, M.A. Chellaiah (2010) Regulation of Erk1/2 activation by osteopontin in PC3 human prostate cancer cells. Mol Cancer 9: 260-270.

Srinivasan, R., B. Chen, J.P. Gorski, A. George (1999) Recombinant expression and characterization of dentin matrix protein 1 . Connect Tissue Res 40: 251-258. 